\title{
The microRNA and p53 families join forces against cancer
}

\author{
NA Barlev ${ }^{*, 1,2}$, BS Sayan ${ }^{3}$, E Candi $^{4}$ and AL Okorokov ${ }^{5}$ \\ Cell Death and Differentiation (2010) 17, 373-375; doi:10.1038/cdd.2009.73
}

p53, microRNA and Cancer, Leicester, UK, 10 December 2008 International mini-simposium held on 10 December in Leicester, UK

The product of the TP53 gene, p53, is one of the most recognised and extensively studied molecules that protects multicellular organisms from cancer. The well-deserved fame of p53 stems from the nature of its function - to coordinate an appropriate cellular response to various forms of genotoxic stress through cell cycle arrest, senescence and/or apoptosis. Given the importance of this role, it is not, perhaps, surprising that p53 was found to be inactive in more than half of human cancers. It should be noted that p53 does not work alone but cooperates with two ancestral proteins of the same family, p63 and $p 73$. Aiming to broaden our understanding of the function of the p53 family of proteins in cancer, the mini-symposium held in the University of Leicester provided an opportunity for scientists and clinicians to exchange knowledge and expertise and to establish new collaborations. The main focus of this event was on the recently emerged link between the p53 family members and microRNA during cancer development.

\section{p53, p63, and MicroRNA}

Recent studies have shown that microRNAs are the components of the p53 and p63 networks, acting both upstream and downstream of these transcription factors. Of these microRNAs, the conserved family of miR-34 seems to be a direct target of p53. However, as highlighted by Nick Barlev, levels of miR-34 expression in different p53-positive cancer cell lines vary significantly, indicating that other miRs contribute to these phenotypes. Barlev's group showed that p53, in addition to activation of the miR-34 locus, both activated and repressed transcription of several additional miRs, promoting the cytotoxic effect of genotoxic drugs. At the molecular level, p53-mediated transcriptional regulation of these miRs involved chromatin remodelling of their respective promoters: H3-K14 acetylation in case of activation and H3-K9/K27 methylation in case of repression. Moreover, DNA damageinduced post-translational modifications of p53 dictated specificity of its interactions with either histone acetyltransferases (HATs) or histone methyltransferases (HMTases) to regulate transcription. Thus, a working model has been suggested whereby the cell type-specific repertoire of miRs regulated by $\mathrm{p} 53$ defines, at least in part, sensitivity of particular tumours to the effect of anti-cancer genotoxic drugs.

In another p53- and miR-34-focused study, Martin Bushell and colleagues suggested the presence of additional mechanisms for regulation of miR-34c expression independent of p53. Two major lines of evidence supporting this statement were presented at the meeting: first, the induction of miR-34c was not dependent on p53 per se, but was rather activated by DNA damage in general. Second, overexpression of p53 alone did not induce a significant increase of miR$34 \mathrm{c}$ levels. Moreover, it has been shown that the $3^{\prime}$ UTR of $c$-myc gene contained a single binding site for miR-34, also conserved in zebrafish and chicken. miR-34c bound primarily to this site and strongly repressed the translation of c-myc mRNA without a decrease in mRNA levels. Accordingly, accumulation of miR-34c as a result of DNA damage by etoposide or adriamycin significantly reduced the intracellular protein level of c-Myc without affecting its mRNA level.

The complex tissue-specific expression patterns of miRs and their prominent role in regulation of gene expression evoke their involvement in the processes of differentiation and development. The talk by Eleonora Candi illuminated the importance of miRs in skin morphogenesis, more specifically in keratinocyte differentiation and proliferation. Candi's group has identified several microRNAs that are modulated during keratinocyte differentiation and proliferation. Among these, miR-203 was found to be induced in primary keratinocytes, concomitant with their differentiation. Furthermore, miR-203 specifically targeted the $3^{\prime}$ UTRs of both human and mouse p63. Overexpression of $\mathrm{miR}-203$ in rapidly proliferating human primary keratinocytes significantly reduced their clonogenic capacity. This result points to miR-203 as a key regulator of the p63-dependent proliferative potential of epithelial precursor cells during keratinocyte differentiation and epithelial development.

\footnotetext{
${ }^{1}$ Department of Biochemistry, University of Leicester, Leicester, UK; ${ }^{2}$ Institute of Cytology, Sankt-Petersburg, Russia; ${ }^{3}$ MRC-Toxicology Unit, University of Leicester, Leicester, UK; ${ }^{4}$ Med. Sperim. and Sci. Biochim., University of Rome, Rome, Italy and ${ }^{5}$ Wolfson Institute for Biomedical Research, University College London, London, UK ${ }^{*}$ Corresponding author: NA Barlev, Department of Biochemistry, University of Leicester, Lancaster Road, Leicester LE1 9HN, UK. 
The talk by Anders Lund emphasised the importance of identification of new miR targets to understand their cellular function better. To identify gene targets of miR-10a, Lund's group used an elegant affinity purification-based approach wherein the miR-10a probe was labelled with biotin for subsequent purification of the miR-10a-mRNA complex on streptavidin-conjugated beads. Using this approach, they found that miRNA miR-10a interacted with the $5^{\prime}$ UTR of mRNAs encoding ribosomal proteins to enhance their translation. Moreover, miR-10a alleviated translational repression of the ribosomal protein mRNAs during amino acid starvation and was required for their translational induction following anisomycin treatment or overexpression of RAS. Taken together, these results suggest that miR-10a may positively control global protein synthesis through the stimulation of ribosomal protein mRNA translation and ribosome biogenesis.

\section{Regulatory Networks of p53 and its Isoforms}

The paradigm of p53 existence in cells as being always represented by only one-sized form of protein has recently been challenged by Jean-Christophe Bourdon and David Lane. They have found that the TP53 gene, like many other genes, can encode at least nine different p53 protein isoforms, whose cellular functions are still enigmatic. Bourdon's group took a closer look at several splice forms of p53 that result in production of the truncated proteins in human breast cancer. One of the isoforms, delta133beta, is lacking the $\mathrm{N}$-terminal transactivation domains as well as the carboxyl terminal regulatory domain. This transcriptionally 'crippled' p53 can only bind DNA through oligomerisation with its full-length sibling but at the expense of blunting the transactivation potential of the latter. Moreover, the delta133beta isoform altered the DNA binding specificity of p53. Therefore, deregulation of $\mathrm{p} 53$ isoforms expression may play an important role in tumour formation, as attenuation of the wild-type p53 response would render the cells more susceptible to further genetic alterations and hence, tumour progression.

Salvador Macip gave insights into the crosstalk between the p53 and retinoic acid (RA) pathways in DNA damage. His group has identified a novel link between the RA and p53 pathways. They found that p53 induces Stra6, the membrane receptor for retinol binding protein (RBP) encoded by a RAresponsive gene after DNA damage, and that this enhances p53-induced apoptosis. Conversely, the absence of Stra6 expression impairs the response of p53 to different stresses. His data suggest that p53 could be engaged in the RA pathway through Stra6 induction to enhance its tumoursuppressor functions. These new data further broaden our understanding of the complexity of p53 regulatory networks.

In addition to p53 and microRNAs, yet another family of proteins, called sirtuins, has emerged recently as a coordinator of stress response in cells. The human orthologue of yeast Sir2, Sirt1, is an NAD + -dependent protein deacetylase that targets both histone and non-histone proteins such as $\mathrm{NF}-\kappa \mathrm{B}, \mathrm{Ku}$ and $\mathrm{p} 53$. Human Sirt1 is involved in differentiation, homoeostasis and metabolism, and is linked to a diverse spectrum of diseases, including cancer. Sirt1 can function as a cancer-specific survival factor. The anti-apoptotic role of Sirt1 is regulated at the levels of transcription, mRNA stability and protein activity. Jo Milner discussed the regulatory interplay between p53 and Sirt1 in cancer. The data presented by her at the meeting suggested that Sirt1 was also regulated at the post-translational level through phosphorylation mediated by a stress-responsive kinase JNK2. JNK2mediated phosphorylation of Sirt1 on Ser-27 led to its stabilisation. Conversely, p53 was involved in destabilisation of Sirt1 mRNA following the treatment of tumour cells with the clinically approved anti-cancer drug 5-fluorouracil (5-FU). Collectively, these results suggest that JNK2 may enhance the cancer survival effect of Sirt1.

\section{Structure Models of p53}

Undoubtedly, restoration of the p53 function in malignant cells would tremendously enhance the efficacy of existing anticancer therapies. Thus, it is imperative to understand the architecture of the p53 protein to facilitate the rational design of small molecule drugs that would efficiently revert the cancer-associated mutants of p53 to their normal state. Such information can be deduced from the p53 structure. Indeed, in 1994, the crystal structure of the central core domain of p53 bound to its specific DNA was elucidated. However, the full-length p53 protein is notoriously flexible conformationally and thus represents an insurmountable challenge for structural biologists. Nevertheless, the structural information on the p53 central core domain has started to pay dividends. Alan Fersht described the work of his group on Y220C mutation in the core domain of $p 53$. This mutation occurs with a frequency of 70000-80 000 new cases annually and has been shown to be highly destabilised at human body temperature. Detailed structural analysis showed that a change on p53's surface resulting from this mutation could be potentially exploited to design small molecules that will bind to and stabilise the protein. Subsequent studies successfully identified such small compounds that selectively bind mutant p53 and restore its activity.

Recent rapid development of structural biophysical methods such as electron microscopy (EM) combined with image processing and small-angle X-ray scattering (SAXS) coupled with computer analysis has provided a powerful tool for analysis of macromolecular complexes in multiple states. On the basis of this analysis, the structure of modified (stabilised) human full-length p53 in complex with DNA has been suggested by Alan Fersht's group. The model suggests that the four core domains of p53 make pairs when bound to DNA, and are linked by flexible linkers to the oligomerisation node. This composition would ensure an overall twofold rotational (C2) symmetry of the p53 molecule in which the oligomerisation domain is located on one side of the structure.

Andrei Okorokov and colleagues have solved the structure of full-length murine p53 using a cryo-EM technique. Comparison of these two models obtained using different experimental approaches showed drastic differences between them. According to the cryo-EM data, the p53 tetramer is more compact and is shaped like a skewed cube. Notably, unlike in the other model, the C-termini of p53 do not interact with each other. Therefore, p53 oligomerisation is mediated 
not through co-assembly of its tetramerisation domains but instead through interactions between the $\mathrm{N}$ - and $\mathrm{C}$-terminal $\alpha$-helices that form a dimerisation interface. Thus, each p53 monomer contains three regions of contact with other subunits of the tetramer. Two of these contacts are provided by reciprocal $\mathrm{N}$ - and $\mathrm{C}$-termini that abut together within a dimer, and the third one is formed between the central core domains of one dimer and the N/C nodes of the second dimer.

Importantly, this model also suggests an alternative mode for p53/DNA interaction. p53 can accommodate variously spaced sequence-specific DNA response elements through flexibility of their dimer + dimer organisation instead of flexible linkers between the cores and C-termini, as suggested by NSEM and SAXS analyses. Numerous biological data seem to favour the cryo-EM-based model. Solving the structures of the p53 ancestral proteins, p63 and p73, should ultimately help to test the validity of each model.

\section{Role of p73 and p63 in Differentiation and Development}

It is becoming more and more apparent that p53 alone would not be able to cope efficiently with the wide spectrum of diverse biological processes controlled by this molecule. Research in the last decade has begun to recognise the importance of p63 and p73 proteins. Gerry Melino talked about their recent findings in the field of $p 73$ function studies. To investigate the precise role of TAp73, a pro-apoptotic isoform of p73 in embryonic development, tumour and cell death, they have generated TAp73 knock-out mice. Interestingly, these mice exhibited an increased incidence of spontaneous tumours because of increased genomic instability associated with aneuploidy. Moreover, these mice were sterile because of a decreased number of ovulated oocytes. This phenomenon can be explained by the fact that some germ cells were retained within the ovary.

Daniel Aberdam gave new and exciting insights into the role of p63 in development. A cellular in vitro ES cells-based model that recapitulates the early embryonic steps of epidermal development was used to study the function of p63. Using this system, Aberdam's group was able to show that p63 is essential for epidermal commitment of ectodermal progenitors. Unexpectedly, p63 was also found to be important in heart development as p63 null mice exhibited severe defects in embryonic cardiac development. One patient affected by an ED syndrome due to a mutation in the $p 63$ gene was reported to have arrhythmogenic right ventricular cardiomyopathy, which resembles the cardiac defect in p63-/- mice. Therefore, some patients affected by p63-linked ED syndromes could be at risk of unexpected heart failure.

To identify the network of p63-regulated genes, Roberto Mantovani's group has used two strategies: (i) gene expression profiling of p63-depleted cells and (ii) chromatin immunoprecipitation coupled to microarray technology (ChIP-on-chip). They have already reported 186 targets of p63 and categorised gene targets of p63 according to their role in biological processes. This analysis linked p63 to cell growth and differentiation as well as stratification of human skin.

\section{Concluding Remarks}

Despite its mini-format, this international meeting gave a succinct update of the wide field of research on the p53 family members and highlighted their importance in biology and medicine. Despite hundreds of papers published in this field every year, many aspects still remain to be investigated. Besides new insights in the molecular functioning of the p53 family members, this meeting emphasised the importance of cross talk between different research fields to solve the fundamental problems of molecular cancer biology. It is the unanimous opinion of participants and attendees that such meetings should be held in future on a regular basis.

Acknowledgements. Grant support to NB from $\mathrm{NIH/NCl,} \mathrm{AICR,} \mathrm{FAMRI}$ and MCB-RAS and to AO from BBSRC and MRC is appreciated. 